\title{
Structured Learning for Taxonomy Induction with Belief Propagation
}

\author{
Mohit Bansal \\ TTI Chicago \\ mbansal@ttic.edu
}

\author{
David Burkett \\ Twitter Inc. \\ dburkett@twitter.com
}

\author{
Gerard de Melo \\ Tsinghua University \\ gdm@demelo.org
}

\author{
Dan Klein \\ UC Berkeley \\ klein@cs.berkeley.edu
}

\begin{abstract}
We present a structured learning approach to inducing hypernym taxonomies using a probabilistic graphical model formulation. Our model incorporates heterogeneous relational evidence about both hypernymy and siblinghood, captured by semantic features based on patterns and statistics from Web $n$-grams and Wikipedia abstracts. For efficient inference over taxonomy structures, we use loopy belief propagation along with a directed spanning tree algorithm for the core hypernymy factor. To train the system, we extract sub-structures of WordNet and discriminatively learn to reproduce them, using adaptive subgradient stochastic optimization. On the task of reproducing sub-hierarchies of WordNet, our approach achieves a $51 \%$ error reduction over a chance baseline, including a $15 \%$ error reduction due to the non-hypernym-factored sibling features. On a comparison setup, we find up to $29 \%$ relative error reduction over previous work on ancestor F1.
\end{abstract}

\section{Introduction}

Many tasks in natural language understanding, such as question answering, information extraction, and textual entailment, benefit from lexical semantic information in the form of types and hypernyms. A recent example is IBM's Jeopardy! system Watson (Ferrucci et al., 2010), which used type information to restrict the set of answer candidates. Information of this sort is present in term taxonomies (e.g., Figure 1), ontologies, and thesauri. However, currently available taxonomies such as WordNet are incomplete in coverage (Pennacchiotti and Pantel, 2006; Hovy et al., 2009), unavailable in many domains and languages, and

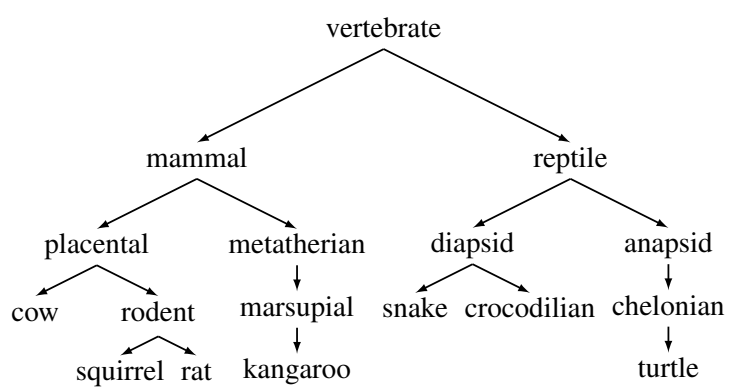

Figure 1: An excerpt of WordNet's vertebrates taxonomy.

time-intensive to create or extend manually. There has thus been considerable interest in building lexical taxonomies automatically.

In this work, we focus on the task of taking collections of terms as input and predicting a complete taxonomy structure over them as output. Our model takes a loglinear form and is represented using a factor graph that includes both 1st-order scoring factors on directed hypernymy edges (a parent and child in the taxonomy) and 2nd-order scoring factors on sibling edge pairs (pairs of hypernym edges with a shared parent), as well as incorporating a global (directed spanning tree) structural constraint. Inference for both learning and decoding uses structured loopy belief propagation (BP), incorporating standard spanning tree algorithms (Chu and Liu, 1965; Edmonds, 1967; Tutte, 1984). The belief propagation approach allows us to efficiently and effectively incorporate heterogeneous relational evidence via hypernymy and siblinghood (e.g., coordination) cues, which we capture by semantic features based on simple surface patterns and statistics from Web $n$-grams and Wikipedia abstracts. We train our model to maximize the likelihood of existing example ontologies using stochastic optimization, automatically learning the most useful relational patterns for full taxonomy induction.

As an example of the relational patterns that our 
system learns, suppose we are interested in building a taxonomy for types of mammals (see Figure 1). Frequent attestation of hypernymy patterns like rat is a rodent in large corpora is a strong signal of the link rodent $\rightarrow$ rat. Moreover, sibling or coordination cues like either rats or squirrels suggest that rat is a sibling of squirrel and adds evidence for the links rodent $\rightarrow$ rat and rodent $\rightarrow$ squirrel. Our supervised model captures exactly these types of intuitions by automatically discovering such heterogeneous relational patterns as features (and learning their weights) on edges and on sibling edge pairs, respectively.

There have been several previous studies on taxonomy induction. e.g., the incremental taxonomy induction system of Snow et al. (2006), the longest path approach of Kozareva and Hovy (2010), and the maximum spanning tree (MST) approach of Navigli et al. (2011) (see Section 4 for a more detailed overview). The main contribution of this work is that we present the first discriminatively trained, structured probabilistic model over the full space of taxonomy trees, using a structured inference procedure through both the learning and decoding phases. Our model is also the first to directly learn relational patterns as part of the process of training an end-to-end taxonomic induction system, rather than using patterns that were hand-selected or learned via pairwise classifiers on manually annotated co-occurrence patterns. Finally, it is the first end-to-end (i.e., nonincremental) system to include sibling (e.g., coordination) patterns at all.

We test our approach in two ways. First, on the task of recreating fragments of WordNet, we achieve a $51 \%$ error reduction on ancestor-based $\mathrm{F} 1$ over a chance baseline, including a $15 \%$ error reduction due to the non-hypernym-factored sibling features. Second, we also compare to the results of Kozareva and Hovy (2010) by predicting the large animal subtree of WordNet. Here, we get up to $29 \%$ relative error reduction on ancestorbased F1. We note that our approach falls at a different point in the space of performance tradeoffs from past work - by producing complete, highly articulated trees, we naturally see a more even balance between precision and recall, while past work generally focused on precision. ${ }^{1}$ To

\footnotetext{
${ }^{1}$ While different applications will value precision and recall differently, and past work was often intentionally precision-focused, it is certainly the case that an ideal solution would maximize both.
}

avoid presumption of a single optimal tradeoff, we also present results for precision-based decoding, where we trade off recall for precision.

\section{Structured Taxonomy Induction}

Given an input term set $\boldsymbol{x}=\left\{x_{1}, x_{2}, \ldots, x_{n}\right\}$, we wish to compute the conditional distribution over taxonomy trees $\boldsymbol{y}$. This distribution $P(\boldsymbol{y} \mid \boldsymbol{x})$ is represented using the graphical model formulation shown in Figure 2. A taxonomy tree $y$ is composed of a set of indicator random variables $y_{i j}$ (circles in Figure 2), where $y_{i j}=$ ON means that $x_{i}$ is the parent of $x_{j}$ in the taxonomy tree (i.e. there exists a directed edge from $x_{i}$ to $x_{j}$ ). One such variable exists for each pair $(i, j)$ with $0 \leq i \leq n, 1 \leq j \leq n$, and $i \neq j .^{2}$

In a factor graph formulation, a set of factors (squares and rectangles in Figure 2) determines the probability of each possible variable assignment. Each factor $F$ has an associated scoring function $\phi_{F}$, with the probability of a total assignment determined by the product of all these scores:

$$
P(\boldsymbol{y} \mid \boldsymbol{x}) \propto \prod_{F} \phi_{F}(\boldsymbol{y})
$$

\subsection{Factor Types}

In the models we present here, there are three types of factors: EDGE factors that score individual edges in the taxonomy tree, SIBLING factors that score pairs of edges with a shared parent, and a global TREE factor that imposes the structural constraint that $\boldsymbol{y}$ form a legal taxonomy tree.

EDGE Factors. For each edge variable $y_{i j}$ in the model, there is a corresponding factor $E_{i j}$ (small blue squares in Figure 2) that depends only on $y_{i j}$. We score each edge by extracting a set of features $\mathbf{f}\left(x_{i}, x_{j}\right)$ and weighting them by the (learned) weight vector $\mathbf{w}$. So, the factor scoring function is:

$$
\phi_{E_{i j}}\left(y_{i j}\right)= \begin{cases}\exp \left(\mathbf{w} \cdot \mathbf{f}\left(x_{i}, x_{j}\right)\right) & y_{i j}=\mathrm{ON} \\ \exp (0)=1 & y_{i j}=\mathrm{OFF}\end{cases}
$$

SiBLING Factors. Our second model also includes factors that permit 2nd-order features looking at terms that are siblings in the taxonomy tree. For each triple $(i, j, k)$ with $i \neq j, i \neq k$, and $j<k,{ }^{3}$ we have a factor $S_{i j k}$ (green rectangles in

\footnotetext{
${ }^{2}$ We assume a special dummy root symbol $x_{0}$.

${ }^{3}$ The ordering of the siblings $x_{j}$ and $x_{k}$ doesn't matter here, so having separate factors for $(i, j, k)$ and $(i, k, j)$ would be redundant.
} 


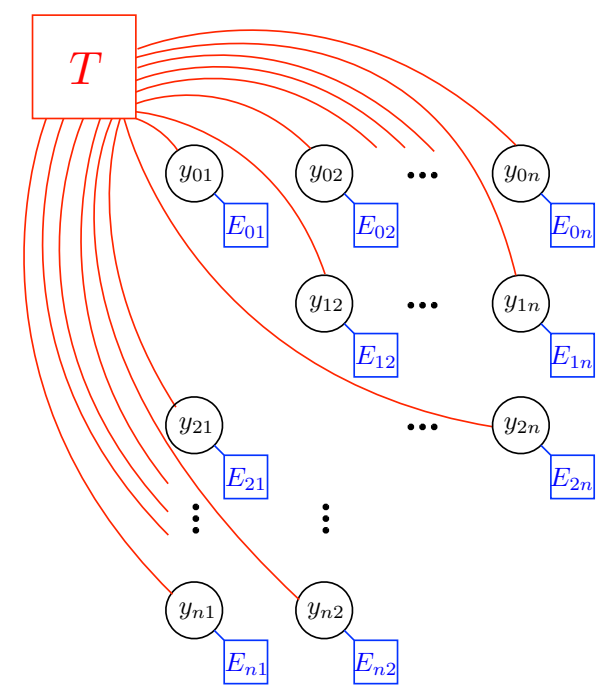

(a) Edge Features Only

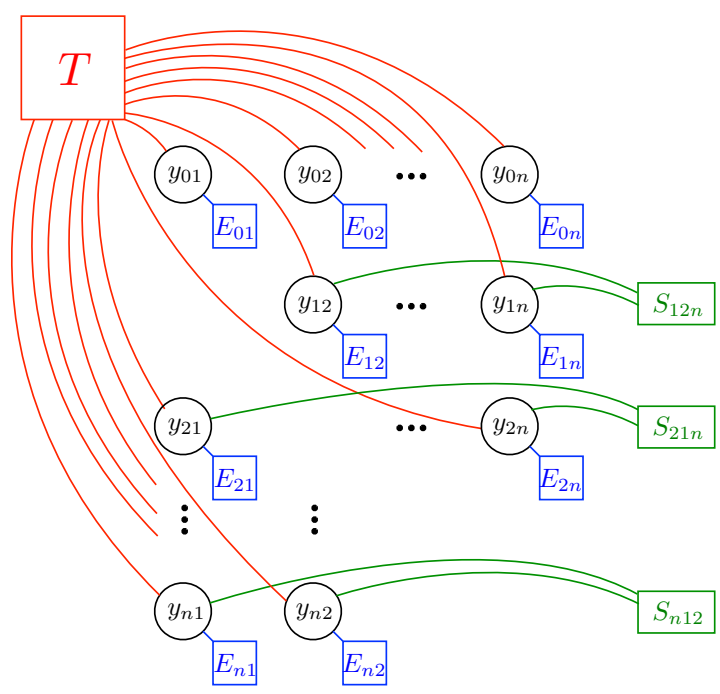

(b) Full Model

Figure 2: Factor graph representation of our model, both without (a) and with (b) SIBLING factors.

Figure $2 \mathrm{~b}$ ) that depends on $y_{i j}$ and $y_{i k}$, and thus can be used to encode features that should be active whenever $x_{j}$ and $x_{k}$ share the same parent, $x_{i}$. The scoring function is similar to the one above:

$\phi_{S_{i j k}}\left(y_{i j}, y_{i k}\right)= \begin{cases}\exp \left(\mathbf{w} \cdot \mathbf{f}\left(x_{i}, x_{j}, x_{k}\right)\right) & y_{i j}=y_{i k}=\mathrm{ON} \\ 1 & \text { otherwise }\end{cases}$

Tree Factor. Of course, not all variable assignments $\boldsymbol{y}$ form legal taxonomy trees (i.e., directed spanning trees). For example, the assignment $\forall i, j, y_{i j}=$ ON might get a high score, but would not be a valid output of the model. Thus, we need to impose a structural constraint to ensure that such illegal variable assignments are assigned 0 probability by the model. We encode this in our factor graph setting using a single global factor $T$ (shown as a large red square in Figure 2) with the following scoring function:

$$
\phi_{T}(\boldsymbol{y})= \begin{cases}1 & \boldsymbol{y} \text { forms a legal taxonomy tree } \\ 0 & \text { otherwise }\end{cases}
$$

Model. For a given global assignment $\boldsymbol{y}$, let

$$
\mathbf{f}(\boldsymbol{y})=\sum_{\substack{i, j \\ y_{i j}=\mathrm{ON}}} \mathbf{f}\left(x_{i}, x_{j}\right)+\sum_{\substack{i, j, k \\ y_{i j}=y_{i k}=\mathrm{ON}}} \mathbf{f}\left(x_{i}, x_{j}, x_{k}\right)
$$

Note that by substituting our model's factor scoring functions into Equation 1, we get:

$$
P(\boldsymbol{y} \mid \boldsymbol{x}) \propto \begin{cases}\exp (\mathbf{w} \cdot \mathbf{f}(\boldsymbol{y})) & \boldsymbol{y} \text { is a tree } \\ 0 & \text { otherwise }\end{cases}
$$

Thus, our model has the form of a standard loglinear model with feature function $\mathbf{f}$.

\subsection{Inference via Belief Propagation}

With the model defined, there are two main inference tasks we wish to accomplish: computing expected feature counts and selecting a particular taxonomy tree for a given set of input terms (decoding). As an initial step to each of these procedures, we wish to compute the marginal probabilities of particular edges (and pairs of edges) being on. In a factor graph, the natural inference procedure for computing marginals is belief propagation. Note that finding taxonomy trees is a structurally identical problem to directed spanning trees (and thereby non-projective dependency parsing), for which belief propagation has previously been worked out in depth (Smith and Eisner, 2008). Therefore, we will only briefly sketch the procedure here.

Belief propagation is a general-purpose inference method that computes marginals via directed messages passed from variables to adjacent factors (and vice versa) in the factor graph. These messages take the form of (possibly unnormalized) distributions over values of the variable. The two types of messages (variable to factor or factor to variable) have mutually recursive definitions. The message from a factor $F$ to an adjacent variable $V$ involves a sum over all possible values of every other variable that $F$ touches. While the EDGE and SIBLING factors are simple enough to compute this sum by brute force, performing the sum naïvely for computing messages from the TREE factor would take exponential time. How- 
ever, due to the structure of that particular factor, all of its outgoing messages can be computed simultaneously in $O\left(n^{3}\right)$ time via an efficient adaptation of Kirchhoff's Matrix Tree Theorem (MTT) (Tutte, 1984) which computes partition functions and marginals for directed spanning trees.

Once message passing is completed, marginal beliefs are computed by merely multiplying together all the messages received by a particular variable or factor.

\subsubsection{Loopy Belief Propagation}

Looking closely at Figure 2a, one can observe that the factor graph for the first version of our model, containing only EDGE and TREE factors, is acyclic. In this special case, belief propagation is exact: after one round of message passing, the beliefs computed (as discussed in Section 2.2) will be the true marginal probabilities under the current model. However, in the full model, shown in Figure 2b, the SIBLING factors introduce cycles into the factor graph, and now the messages being passed around often depend on each other and so they will change as they are recomputed. The process of iteratively recomputing messages based on earlier messages is known as loopy belief propagation. This procedure only finds approximate marginal beliefs, and is not actually guaranteed to converge, but in practice can be quite effective for finding workable marginals in models for which exact inference is intractable, as is the case here. All else equal, the more rounds of message passing that are performed, the closer the computed marginal beliefs will be to the true marginals, though in practice, there are usually diminishing returns after the first few iterations. In our experiments, we used a fairly conservative upper bound of 20 iterations, but in most cases, the messages converged much earlier than that.

\subsection{Training}

We used gradient-based maximum likelihood training to learn the model parameters $\mathbf{w}$. Since our model has a loglinear form, the derivative of $\mathbf{w}$ with respect to the likelihood objective is computed by just taking the gold feature vector and subtracting the vector of expected feature counts. For computing expected counts, we run belief propagation until completion and then, for each factor in the model, we simply read off the marginal probability of that factor being active (as computed in Section 2.2), and accumulate a par- tial count for each feature that is fired by that factor. This method of computing the gradient can be incorporated into any gradient-based optimizer in order to learn the weights $\mathbf{w}$. In our experiments we used AdaGrad (Duchi et al., 2011), an adaptive subgradient variant of standard stochastic gradient ascent for online learning.

\subsection{Decoding}

Finally, once the model parameters have been learned, we want to use the model to find taxonomy trees for particular sets of input terms. Note that if we limit our scores to be edge-factored, then finding the highest scoring taxonomy tree becomes an instance of the MST problem (also known as the maximum arborescence problem for the directed case), which can be solved efficiently in $O\left(n^{2}\right)$ quadratic time (Tarjan, 1977) using the greedy, recursive Chu-Liu-Edmonds algorithm (Chu and Liu, 1965; Edmonds, 1967). ${ }^{4}$

Since the MST problem can be solved efficiently, the main challenge becomes finding a way to ensure that our scores are edge-factored. In the first version of our model, we could simply set the score of each edge to be $\mathbf{w} \cdot \mathbf{f}\left(x_{i}, x_{j}\right)$, and the MST recovered in this way would indeed be the highest scoring tree: $\arg \max _{\boldsymbol{y}} P(\boldsymbol{y} \mid \boldsymbol{x})$. However, this straightforward approach doesn't apply to the full model which also uses sibling features. Hence, at decoding time, we instead start out by once more using belief propagation to find marginal beliefs, and then set the score of each edge to be its belief odds ratio: $\frac{b_{Y_{i j}}(\mathrm{ON})}{b_{Y_{i j}}(\mathrm{OFF})}$.

\section{Features}

While spanning trees are familiar from nonprojective dependency parsing, features based on the linear order of the words or on lexical identi-

\footnotetext{
${ }^{4}$ See Georgiadis (2003) for a detailed algorithmic proof, and McDonald et al. (2005) for an illustrative example. Also, we constrain the Chu-Liu-Edmonds MST algorithm to output only single-root MSTs, where the (dummy) root has exactly one child (Koo et al., 2007), because multi-root spanning 'forests' are not applicable to our task.

Also, note that we currently assume one node per term. We are following the task description from previous work where the goal is to create a taxonomy for a specific domain (e.g., animals). Within a specific domain, terms typically just have a single sense. However, our algorithms could certainly be adapted to the case of multiple term senses (by treating the different senses as unique nodes in the tree) in future work.

${ }^{5}$ The MST that is found using these edge scores is actually the minimum Bayes risk tree (Goodman, 1996) for an edge accuracy loss function (Smith and Eisner, 2008).
} 
ties or syntactic word classes, which are primary drivers for dependency parsing, are mostly uninformative for taxonomy induction. Instead, inducing taxonomies requires world knowledge to capture the semantic relations between various unseen terms. For this, we use semantic cues to hypernymy and siblinghood via features on simple surface patterns and statistics in large text corpora. We fire features on both the edge and the sibling factors. We first describe all the edge features in detail (Section 3.1 and Section 3.2), and then briefly describe the sibling features (Section 3.3), which are quite similar to the edge ones.

For each edge factor $E_{i j}$, which represents the potential parent-child term pair $\left(x_{i}, x_{j}\right)$, we add the surface and semantic features discussed below. Note that since edges are directed, we have separate features for the factors $E_{i j}$ versus $E_{j i}$.

\subsection{Surface Features}

Capitalization: Checks which of $x_{i}$ and $x_{j}$ are capitalized, with one feature for each value of the tuple (isCap $\left(x_{i}\right)$, isCap $\left(x_{j}\right)$ ). The intuition is that leaves of a taxonomy are often proper names and hence capitalized, e.g., (bison, American bison). Therefore, the feature for (true, false) (i.e., parent capitalized but not the child) gets a substantially negative weight.

Ends with: Checks if $x_{j}$ ends with $x_{i}$, or not. This captures pairs such as (fish, bony fish) in our data.

Contains: Checks if $x_{j}$ contains $x_{i}$, or not. This captures pairs such as (bird, bird of prey).

Suffix match: Checks whether the $k$-length suffixes of $x_{i}$ and $x_{j}$ match, or not, for $k=$ $1,2, \ldots, 7$.

LCS: We compute the longest common substring of $x_{i}$ and $x_{j}$, and create indicator features for rounded-off and binned values of $|L C S| /\left(\left(\left|x_{i}\right|+\right.\right.$ $\left.\left|x_{j}\right|\right) / 2$ ).

Length difference: We compute the signed length difference between $x_{j}$ and $x_{i}$, and create indicator features for rounded-off and binned values of $\left(\left|x_{j}\right|-\left|x_{i}\right|\right) /\left(\left(\left|x_{i}\right|+\left|x_{j}\right|\right) / 2\right)$. Yang and Callan (2009) use a similar feature.

\subsection{Semantic Features}

\subsubsection{Web $n$-gram Features}

Patterns and counts: Hypernymy for a term pair $\left(P=x_{i}, C=x_{j}\right)$ is often signaled by the presence of surface patterns like $C$ is a $P, P$ such as $C$ in large text corpora, an observation going back to Hearst (1992). For each potential parent-child edge $\left(P=x_{i}, C=x_{j}\right)$, we mine the top $k$ strings (based on count) in which both $x_{i}$ and $x_{j}$ occur (we use $k=200$ ). We collect patterns in both directions, which allows us to judge the correct direction of an edge (e.g., $C$ is a $P$ is a positive signal for hypernymy whereas $P$ is $a C$ is a negative signal). ${ }^{6}$ Next, for each pattern in this top- $k$ list, we compute its normalized pattern count $c$, and fire an indicator feature on the tuple (pattern, $t$ ), for all thresholds $t$ (in a fixed set) s.t. $c \geq t$. Our supervised model then automatically learns which patterns are good indicators of hypernymy.

Pattern order: We add features on the order (direction) in which the pair $\left(x_{i}, x_{j}\right)$ found a pattern (in its top- $k$ list) - indicator features for boolean values of the four cases: $P \ldots C, C \ldots P$, neither direction, and both directions. Ritter et al. (2009) used the 'both' case of this feature.

Individual counts: We also compute the individual Web-scale term counts $c_{x_{i}}$ and $c_{x_{j}}$, and add a comparison feature $\left(c_{x_{i}}>c_{x_{j}}\right)$, plus features on values of the signed count difference $\left(\left|c_{x_{i}}\right|-\right.$ $\left.\left|c_{x_{j}}\right|\right) /\left(\left(\left|c_{x_{i}}\right|+\left|c_{x_{j}}\right|\right) / 2\right)$, after rounding off, and binning at multiple granularities. The intuition is that this feature could learn whether the relative popularity of the terms signals their hypernymy direction.

\subsubsection{Wikipedia Abstract Features}

The Web $n$-grams corpus has broad coverage but is limited to up to 5 -grams, so it may not contain pattern-based evidence for various longer multiword terms and pairs. Therefore, we supplement it with a full-sentence resource, namely Wikipedia abstracts, which are concise descriptions (hence useful to signal hypernymy) of a large variety of world entities.

Presence and distance: For each potential edge $\left(x_{i}, x_{j}\right)$, we mine patterns from all abstracts in which the two terms co-occur in either order, allowing a maximum term distance of 20 (because beyond that, co-occurrence may not imply a relation). We add a presence feature based on whether the process above found at least one pattern for that term pair, or not. We also fire features on the value of the minimum distance $d_{\min }$ at which

\footnotetext{
${ }^{6}$ We also allow patterns with surrounding words, e.g., the $C$ is a $P$ and $C, P$ of.
} 
the two terms were found in some abstract (plus thresholded versions).

Patterns: For each term pair, we take the top- $k^{\prime}$ patterns (based on count) of length up to $l$ from its full list of patterns, and add an indicator feature on each pattern string (without the counts). We use $k^{\prime}=5, l=10$. Similar to the Web $n$-grams case, we also fire Wikipedia-based pattern order features.

\subsection{Sibling Features}

We also incorporate similar features on sibling factors. For each sibling factor $S_{i j k}$ which represents the potential parent-children term triple $\left(x_{i}, x_{j}, x_{k}\right)$, we consider the potential sibling term pair $\left(x_{j}, x_{k}\right)$. Siblinghood for this pair would be indicated by the presence of surface patterns such as either $C_{1}$ or $C_{2}, C_{1}$ is similar to $C_{2}$ in large corpora. Hence, we fire Web $n$-gram pattern features and Wikipedia presence, distance, and pattern features, similar to those described above, on each potential sibling term pair. ${ }^{7}$ The main difference here from the edge factors is that the sibling factors are symmetric (in the sense that $S_{i j k}$ is redundant to $S_{i k j}$ ) and hence the patterns are undirected. Therefore, for each term pair, we first symmetrize the collected Web $n$-grams and Wikipedia patterns by accumulating the counts of symmetric patterns like rats or squirrels and squirrels or rats. ${ }^{8}$

\section{Related Work}

In our work, we assume a known term set and do not address the problem of extracting related terms from text. However, a great deal of past work has considered automating this process, typically taking one of two major approaches. The clustering-based approach (Lin, 1998; Lin and Pantel, 2002; Davidov and Rappoport, 2006; Yamada et al., 2009) discovers relations based on the assumption that similar concepts appear in sim-

\footnotetext{
${ }^{7}$ One can also add features on the full triple $\left(x_{i}, x_{j}, x_{k}\right)$ but most such features will be sparse.

${ }^{8}$ All the patterns and counts for our Web and Wikipedia edge and sibling features described above are extracted after stemming the words in the terms, the $n$-grams, and the abstracts (using the Porter stemmer). Also, we threshold the features (to prune away the sparse ones) by considering only those that fire for at least $t$ trees in the training data $(t=4$ in our experiments).

Note that one could also add various complementary types of useful features presented by previous work, e.g., bootstrapping using syntactic heuristics (Phillips and Riloff, 2002), dependency patterns (Snow et al., 2006), doubly anchored patterns (Kozareva et al., 2008; Hovy et al., 2009), and Web definition classifiers (Navigli et al., 2011).
}

ilar contexts (Harris, 1954). The pattern-based approach uses special lexico-syntactic patterns to extract pairwise relation lists (Phillips and Riloff, 2002; Girju et al., 2003; Pantel and Pennacchiotti, 2006; Suchanek et al., 2007; Ritter et al., 2009; Hovy et al., 2009; Baroni et al., 2010; Ponzetto and Strube, 2011) and semantic classes or classinstance pairs (Riloff and Shepherd, 1997; Katz and Lin, 2003; Paşca, 2004; Etzioni et al., 2005; Talukdar et al., 2008).

We focus on the second step of taxonomy induction, namely the structured organization of terms into a complete and coherent tree-like hierarchy. ${ }^{9}$ Early work on this task assumes a starting partial taxonomy and inserts missing terms into it. Widdows (2003) place unknown words into a region with the most semantically-similar neighbors. Snow et al. (2006) add novel terms by greedily maximizing the conditional probability of a set of relational evidence given a taxonomy. Yang and Callan (2009) incrementally cluster terms based on a pairwise semantic distance. Lao et al. (2012) extend a knowledge base using a random walk model to learn binary relational inference rules.

However, the task of inducing full taxonomies without assuming a substantial initial partial taxonomy is relatively less well studied. There is some prior work on the related task of hierarchical clustering, or grouping together of semantically related words (Cimiano et al., 2005; Cimiano and Staab, 2005; Poon and Domingos, 2010; Fountain and Lapata, 2012). The task we focus on, though, is the discovery of direct taxonomic relationships (e.g., hypernymy) between words.

We know of two closely-related previous systems, Kozareva and Hovy (2010) and Navigli et al. (2011), that build full taxonomies from scratch. Both of these systems use a process that starts by finding basic level terms (leaves of the final taxonomy tree, typically) and then using relational patterns (hand-selected ones in the case of Kozareva and Hovy (2010), and ones learned separately by a pairwise classifier on manually annotated co-occurrence patterns for Navigli and Velardi (2010), Navigli et al. (2011)) to find intermediate terms and all the attested hypernymy links between them. ${ }^{10}$ To prune down the resulting tax-

\footnotetext{
${ }^{9}$ Determining the set of input terms is orthogonal to our work, and our method can be used in conjunction with various term extraction approaches described above.

${ }^{10}$ Unlike our system, which assumes a complete set of terms and only attempts to induce the taxonomic structure,
} 
onomy graph, Kozareva and Hovy (2010) use a procedure that iteratively retains the longest paths between root and leaf terms, removing conflicting graph edges as they go. The end result is acyclic, though not necessarily a tree; Navigli et al. (2011) instead use the longest path intuition to weight edges in the graph and then find the highest weight taxonomic tree using a standard MST algorithm.

Our work differs from the two systems above in that ours is the first discriminatively trained, structured probabilistic model over the full space of taxonomy trees that uses structured inference via spanning tree algorithms (MST and MTT) through both the learning and decoding phases. Our model also automatically learns relational patterns as a part of the taxonomic training phase, instead of relying on hand-picked rules or pairwise classifiers on manually annotated co-occurrence patterns, and it is the first end-to-end (i.e., nonincremental) system to include heterogeneous relational information via sibling (e.g., coordination) patterns.

\section{Experiments}

\subsection{Data and Experimental Regime}

We considered two distinct experimental setups, one that illustrates the general performance of our model by reproducing various medium-sized WordNet domains, and another that facilitates comparison to previous work by reproducing the much larger animal subtree provided by Kozareva and Hovy (2010).

General setup: In order to test the accuracy of structured prediction on medium-sized fulldomain taxonomies, we extracted from WordNet 3.0 all bottomed-out full subtrees which had a tree-height of 3 (i.e., 4 nodes from root to leaf), and contained $(10,50]$ terms. ${ }^{11}$ This gives us 761 non-overlapping trees, which we partition into

both these systems include term discovery in the taxonomy building process.

${ }^{11}$ Subtrees that had a smaller or larger tree height were discarded in order to avoid overlap between the training and test divisions. This makes it a much stricter setting than other tasks such as parsing, which usually has repeated sentences, clauses and phrases between training and test sets.

To project WordNet synsets to terms, we used the first (most frequent) term in each synset. A few WordNet synsets have multiple parents so we only keep the first of each such pair of overlapping trees. We also discard a few trees with duplicate terms because this is mostly due to the projection of different synsets to the same term, and theoretically makes the tree a graph.
70/15/15\% (533/114/114 trees) train/dev/test sets.

Comparison setup: We also compare our method (as closely as possible) with related previous work by testing on the much larger animal subtree made available by Kozareva and Hovy (2010), who created this dataset by selecting a set of 'harvested' terms and retrieving all the WordNet hypernyms between each input term and the root (i.e., animal), resulting in $\sim 700$ terms and $\sim 4,300$ is-a ancestor-child links. ${ }^{12}$ Our training set for this animal test case was generated from WordNet using the following process: First, we strictly remove the full animal subtree from WordNet in order to avoid any possible overlap with the test data. Next, we create random 25 -sized trees by picking random nodes as singleton trees, and repeatedly adding child edges from WordNet to the tree. This process gives us a total of $\sim 1600$ training trees. ${ }^{13}$

Feature sources: The $n$-gram semantic features are extracted from the Google $n$-grams corpus (Brants and Franz, 2006), a large collection of English $n$-grams (for $n=1$ to 5) and their frequencies computed from almost 1 trillion tokens (95 billion sentences) of Web text. The Wikipedia abstracts are obtained via the publicly available dump, which contains almost $\sim 4.1$ million articles. $^{14}$ Preprocessing includes standard XML parsing and tokenization. Efficient collection of feature statistics is important because these must be extracted for millions of query pairs (for each potential edge and sibling pair in each term set). For this, we use a hash-trie on term pairs (similar to that of Bansal and Klein (2011)), and scan once through the $n$-gram (or abstract) set, skipping many $n$-grams (or abstracts) based on fast checks of missing unigrams, exceeding length, suffix mismatches, etc.

\subsection{Evaluation Metric}

Ancestor F1: Measures the precision, recall, and $F_{1}=2 P R /(P+R)$ of correctly predicted ances-

\footnotetext{
${ }^{12}$ This is somewhat different from our general setup where we work with any given set of terms; they start with a large set of leaves which have substantial Web-based relational information based on their selected, hand-picked patterns. Their data is available at http://www.isi.edu/ kozareva/ downloads.html.

${ }^{13}$ We tried this training regimen as different from that of the general setup (which contains only bottomed-out subtrees), so as to match the animal test tree, which is of depth 12 and has intermediate nodes from higher up in WordNet.

${ }^{14}$ We used the 20130102 dump.
} 


\begin{tabular}{|l|ccc|}
\hline System & P & R & F1 \\
\hline \hline \multicolumn{3}{|c|}{ Edges-Only } & Model \\
\hline \hline Baseline & 5.9 & 8.3 & 6.9 \\
Surface Features & 17.5 & 41.3 & 24.6 \\
Semantic Features & 37.0 & 49.1 & 42.2 \\
Surface+Semantic & 41.1 & 54.4 & 46.8 \\
\hline \hline \multicolumn{3}{|c|}{ Edges + Siblings Model } \\
\hline \hline Surface+Semantic & $\mathbf{5 3 . 1}$ & $\mathbf{5 6 . 6}$ & $\mathbf{5 4 . 8}$ \\
\hline Surface+Semantic (Test) & 48.0 & 55.2 & 51.4 \\
\hline
\end{tabular}

Table 1: Main results on our general setup. On the development set, we present incremental results on the edges-only model where we start with the chance baseline, then use surface features only, semantic features only, and both. Finally, we add sibling factors and features to get results for the full, edges+siblings model with all features, and also report the final test result for this setting.

tors, i.e., pairwise $i s-a$ relations:

$P=\frac{\mid \text { isagold }_{\text {gis }} \cap \text { isa }_{\text {predicted }} \mid}{\mid \text { isa }_{\text {predicted }} \mid}, R=\frac{\mid \text { isa }_{\text {gold }} \cap \text { isa }_{\text {predicted }} \mid}{\mid \text { isa }_{\text {gold }} \mid}$

\subsection{Results}

Table 1 shows our main results for ancestor-based evaluation on the general setup. We present a development set ablation study where we start with the edges-only model (Figure 2a) and its random tree baseline (which chooses any arbitrary spanning tree for the term set). Next, we show results on the edges-only model with surface features (Section 3.1), semantic features (Section 3.2), and both. We see that both surface and semantic features make substantial contributions, and they also stack. Finally, we add the sibling factors and features (Figure 2b, Section 3.3), which further improves the results significantly $(8 \%$ absolute and $15 \%$ relative error reduction over the edges-only results on the ancestor F1 metric). The last row shows the final test set results for the full model with all features.

Table 2 shows our results for comparison to the larger animal dataset of Kozareva and Hovy (2010). ${ }^{15}$ In the table, 'Kozareva2010' refers to Kozareva and Hovy (2010) and 'Navigli2011' refers to Navigli et al. (2011). ${ }^{16}$ For appropri-

\footnotetext{
${ }^{15}$ These results are for the 1st order model due to the scale of the animal taxonomy ( $\sim 700$ terms). For scaling the 2 nd order sibling model, one can use approximations, e.g., pruning the set of sibling factors based on 1st order link marginals, or a hierarchical coarse-to-fine approach based on taxonomy induction on subtrees, or a greedy approach of adding a few sibling factors at a time. This is future work.

${ }^{16}$ The Kozareva and Hovy (2010) ancestor results are obtained by using the output files provided on their webpage.
}

\begin{tabular}{|l|ccc|}
\hline System & $\mathrm{P}$ & $\mathrm{R}$ & $\mathrm{F} 1$ \\
\hline \hline \multicolumn{4}{|c|}{ Previous Work } \\
\hline \hline Kozareva2010 & $\mathbf{9 8 . 6}$ & 36.2 & 52.9 \\
Navigli2011 & $97.0^{\star \star}$ & $43.7^{\star \star}$ & $60.3^{\star \star}$ \\
\hline \hline \multicolumn{4}{|c|}{ This Paper } \\
\hline \hline Fixed Prediction & 84.2 & $\mathbf{5 5 . 1}$ & $\mathbf{6 6 . 6}$ \\
Free Prediction & 79.3 & 49.0 & 60.6 \\
\hline
\end{tabular}

Table 2: Comparison results on the animal dataset of Kozareva and Hovy (2010). Here, 'Kozareva2010' refers to Kozareva and Hovy (2010) and 'Navigli2011' refers to Navigli et al. (2011). For appropriate comparison to each previous work, we show our results both for the 'Fixed Prediction' setup, which assumes the true root and leaves, and for the 'Free Prediction' setup, which doesn't assume any prior information. The $\star \star$ results of Navigli et al. (2011) represent a different ground-truth data condition, making them incomparable to our results; see Section 5.3 for details.

ate comparison to each previous work, we show results for two different setups. The first setup 'Fixed Prediction' assumes that the model knows the true root and leaves of the taxonomy to provide for a somewhat fairer comparison to Kozareva and Hovy (2010). We get substantial improvements on ancestor-based recall and F1 (a 29\% relative error reduction). The second setup 'Free Prediction' assumes no prior knowledge and predicts the full tree (similar to the general setup case). On this setup, we do compare as closely as possible to Navigli et al. (2011) and see a small gain in F1, but regardless, we should note that their results are incomparable (denoted by $\star \star$ in Table 2 ) because they have a different ground-truth data condition: their definition and hypernym extraction phase involves using the Google def ine keyword, which often returns WordNet glosses itself.

We note that previous work achieves higher ancestor precision, while our approach achieves a more even balance between precision and recall. Of course, precision and recall should both ideally be high, even if some applications weigh one over the other. This is why our tuning optimized for $F 1$, which represents a neutral combination for comparison, but other $F_{\alpha}$ metrics could also be optimized. In this direction, we also tried an experiment on precision-based decoding (for the 'Free Prediction' scenario), where we discard any edges with score (i.e., the belief odds ratio described in Section 2.4) less than a certain threshold. This allowed us to achieve high values of precision (e.g., 90.8\%) at still high enough F1 values (e.g., 61.7\%). 


\begin{tabular}{|c|c|}
\hline \multicolumn{2}{|c|}{ Hypernymy features } \\
\hline$C$ and other $P$ & $>P>C$ \\
\hline$C, P$ of & $C$ is a $P$ \\
\hline$C, a P$ & $P$, including $C$ \\
\hline C or other $P$ & $P(C$ \\
\hline$C: a P$ & $C$, american $P$ \\
\hline$C$ - like $P$ & $C$, the $P$ \\
\hline \multicolumn{2}{|c|}{ Siblinghood features } \\
\hline " $C_{1}$ and $C_{2}$ & $C_{1}, C_{2}($ \\
\hline$C_{1}$ or $C_{2}$ of & $C_{1}$ and / or $C_{2}$ \\
\hline , $C_{1}, C_{2}$ and & either $C_{1}$ or $C_{2}$ \\
\hline the $C_{1} / C_{2}$ & $<s>C_{1}$ and $C_{2}</ s>$ \\
\hline
\end{tabular}

Table 3: Examples of high-weighted hypernymy and siblinghood features learned during development.

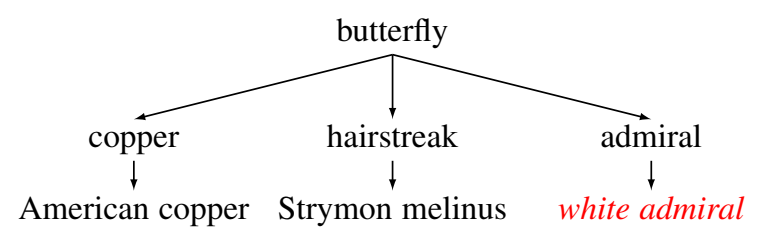

Figure 3: Excerpt from the predicted butterfly tree. The terms attached erroneously according to WordNet are marked in red and italicized.

\section{Analysis}

Table 3 shows some of the hypernymy and siblinghood features given highest weight by our model (in general-setup development experiments). The training process not only rediscovers most of the standard Hearst-style hypernymy patterns (e.g., $C$ and other $P, C$ is a $P$ ), but also finds various novel, intuitive patterns. For example, the pattern $C$, american $P$ is prominent because it captures pairs like Lemmon, american actor and Bryon, american politician, etc. Another pattern $>P>$ $C$ captures webpage navigation breadcrumb trails (representing category hierarchies). Similarly, the algorithm also discovers useful siblinghood features, e.g., either $C_{1}$ or $C_{2}, C_{1}$ and / or $C_{2}$, etc.

Finally, we look at some specific output errors to give as concrete a sense as possible of some system confusions, though of course any hand-chosen examples must be taken as illustrative. In Figure 3 , we attach white admiral to admiral, whereas the gold standard makes these two terms siblings. In reality, however, white admirals are indeed a species of admirals, so WordNet's ground truth turns out to be incomplete. Another such example is that we place logistic assessment in the evalu-

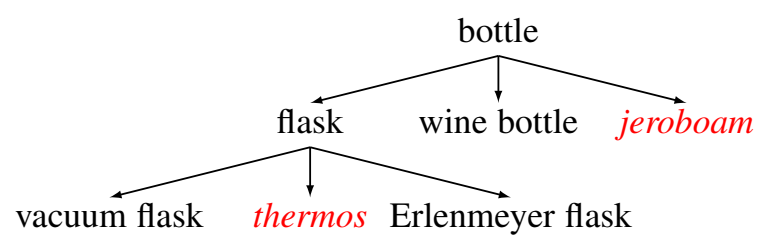

Figure 4: Excerpt from the predicted bottle tree. The terms attached erroneously according to WordNet are marked in red and italicized.

ation subtree of judgment, but WordNet makes it a direct child of judgment. However, other dictionaries do consider logistic assessments to be evaluations. Hence, this illustrates that there may be more than one right answer, and that the low results on this task should only be interpreted as such. In Figure 4, our algorithm did not recognize that thermos is a hyponym of vacuum flask, and that jeroboam is a kind of wine bottle. Here, our Web $n$-grams dataset (which only contains frequent $n$-grams) and Wikipedia abstracts do not suffice and we would need to add richer Web data for such world knowledge to be reflected in the features.

\section{Conclusion}

Our approach to taxonomy induction allows heterogeneous information sources to be combined and balanced in an error-driven way. Direct indicators of hypernymy, such as Hearst-style context patterns, are the core feature for the model and are discovered automatically via discriminative training. However, other indicators, such as coordination cues, can indicate that two words might be siblings, independently of what their shared parent might be. Adding second-order factors to our model allows these two kinds of evidence to be weighed and balanced in a discriminative, structured probabilistic framework. Empirically, we see substantial gains (in ancestor F1) from sibling features, and also over comparable previous work. We also present results on the precision and recall trade-offs inherent in this task.

\section{Acknowledgments}

We would like to thank the anonymous reviewers for their insightful comments. This work was supported by BBN under DARPA contract HR0011-12-C-0014, 973 Program China Grants 2011CBA00300, 2011CBA00301, and NSFC Grants 61033001, 61361136003. 


\section{References}

Mohit Bansal and Dan Klein. 2011. Web-scale features for full-scale parsing. In Proceedings of ACL.

Marco Baroni, Brian Murphy, Eduard Barbu, and Massimo Poesio. 2010. Strudel: A corpus-based semantic model based on properties and types. Cognitive Science, 34(2):222-254.

Thorsten Brants and Alex Franz. 2006. The Google Web 1T 5-gram corpus version 1.1. LDC2006T13.

Yoeng-Jin Chu and Tseng-Hong Liu. 1965. On the shortest arborescence of a directed graph. Science Sinica, 14(1396-1400):270.

Philipp Cimiano and Steffen Staab. 2005. Learning concept hierarchies from text with a guided agglomerative clustering algorithm. In Proceedings of the ICML 2005 Workshop on Learning and Extending Lexical Ontologies with Machine Learning Methods.

Philipp Cimiano, Andreas Hotho, and Steffen Staab. 2005. Learning concept hierarchies from text corpora using formal concept analysis. Journal of Artificial Intelligence Research, 24(1):305-339.

Dmitry Davidov and Ari Rappoport. 2006. Efficient unsupervised discovery of word categories using symmetric patterns and high frequency words. In Proceedings of COLING-ACL.

John Duchi, Elad Hazan, and Yoram Singer. 2011. Adaptive subgradient methods for online learning and stochastic optimization. The Journal of $\mathrm{Ma}$ chine Learning Research, 12:2121-2159.

Jack Edmonds. 1967. Optimum branchings. Journal of Research of the National Bureau of Standards B, 71:233-240.

Oren Etzioni, Michael Cafarella, Doug Downey, AnaMaria Popescu, Tal Shaked, Stephen Soderland, Daniel S. Weld, and Alexander Yates. 2005. Unsupervised named-entity extraction from the Web: An experimental study. Artificial Intelligence, 165(1):91-134.

David Ferrucci, Eric Brown, Jennifer Chu-Carroll, James Fan, David Gondek, Aditya A Kalyanpur, Adam Lally, J William Murdock, Eric Nyberg, John Prager, Nico Schlaefer, and Chris Welty. 2010. Building watson: An overview of the DeepQA project. AI magazine, 31(3):59-79.

Trevor Fountain and Mirella Lapata. 2012. Taxonomy induction using hierarchical random graphs. In Proceedings of NAACL.

Leonidas Georgiadis. 2003. Arborescence optimization problems solvable by edmonds algorithm. Theoretical Computer Science, 301(1):427-437.
Roxana Girju, Adriana Badulescu, and Dan Moldovan. 2003. Learning semantic constraints for the automatic discovery of part-whole relations. In Proceedings of NAACL.

Joshua Goodman. 1996. Parsing algorithms and metrics. In Proceedings of $A C L$.

Zellig Harris. 1954. Distributional structure. Word, 10(23):146-162.

Marti Hearst. 1992. Automatic acquisition of hyponyms from large text corpora. In Proceedings of COLING.

Eduard Hovy, Zornitsa Kozareva, and Ellen Riloff. 2009. Toward completeness in concept extraction and classification. In Proceedings of EMNLP.

Boris Katz and Jimmy Lin. 2003. Selectively using relations to improve precision in question answering. In Proceedings of the Workshop on NLP for Question Answering (EACL 2003).

Terry Koo, Amir Globerson, Xavier Carreras, and Michael Collins. 2007. Structured prediction models via the matrix-tree theorem. In Proceedings of EMNLP-CoNLL.

Zornitsa Kozareva and Eduard Hovy. 2010. A semi-supervised method to learn and construct taxonomies using the Web. In Proceedings of EMNLP.

Zornitsa Kozareva, Ellen Riloff, and Eduard Hovy. 2008. Semantic class learning from the web with hyponym pattern linkage graphs. In Proceedings of $A C L$.

Ni Lao, Amarnag Subramanya, Fernando Pereira, and William W. Cohen. 2012. Reading the web with learned syntactic-semantic inference rules. In Proceedings of EMNLP.

Dekang Lin and Patrick Pantel. 2002. Concept discovery from text. In Proceedings of COLING.

Dekang Lin. 1998. Automatic retrieval and clustering of similar words. In Proceedings of COLING.

Ryan McDonald, Fernando Pereira, Kiril Ribarov, and Jan Hajič. 2005. Non-projective dependency parsing using spanning tree algorithms. In Proceedings of HLT-EMNLP.

Roberto Navigli and Paola Velardi. 2010. Learning word-class lattices for definition and hypernym extraction. In Proceedings of the 48th Annual Meeting of the Association for Computational Linguistics.

Roberto Navigli, Paola Velardi, and Stefano Faralli. 2011. A graph-based algorithm for inducing lexical taxonomies from scratch. In Proceedings of IJCAI.

Patrick Pantel and Marco Pennacchiotti. 2006. Espresso: Leveraging generic patterns for automatically harvesting semantic relations. In Proceedings of COLING-ACL. 
Marius Paşca. 2004. Acquisition of categorized named entities for web search. In Proceedings of CIKM.

Marco Pennacchiotti and Patrick Pantel. 2006. Ontologizing semantic relations. In Proceedings of COLING-ACL.

William Phillips and Ellen Riloff. 2002. Exploiting strong syntactic heuristics and co-training to learn semantic lexicons. In Proceedings of EMNLP.

Simone Paolo Ponzetto and Michael Strube. 2011. Taxonomy induction based on a collaboratively built knowledge repository. Artificial Intelligence, 175(9):1737-1756.

Hoifung Poon and Pedro Domingos. 2010. Unsupervised ontology induction from text. In Proceedings of $A C L$.

Ellen Riloff and Jessica Shepherd. 1997. A corpusbased approach for building semantic lexicons. In Proceedings of EMNLP.

Alan Ritter, Stephen Soderland, and Oren Etzioni. 2009. What is this, anyway: Automatic hypernym discovery. In Proceedings of AAAI Spring Symposium on Learning by Reading and Learning to Read.

David A. Smith and Jason Eisner. 2008. Dependency parsing by belief propagation. In Proceedings of EMNLP.

Rion Snow, Daniel Jurafsky, and Andrew Y. Ng. 2006. Semantic taxonomy induction from heterogenous evidence. In Proceedings of COLING-ACL.

Fabian M. Suchanek, Gjergji Kasneci, and Gerhard Weikum. 2007. Yago: a core of semantic knowledge. In Proceedings of $W W W$.

Partha Pratim Talukdar, Joseph Reisinger, Marius Paşca, Deepak Ravichandran, Rahul Bhagat, and Fernando Pereira. 2008. Weakly-supervised acquisition of labeled class instances using graph random walks. In Proceedings of EMNLP.

Robert E. Tarjan. 1977. Finding optimum branchings. Networks, 7:25-35.

William T. Tutte. 1984. Graph theory. AddisonWesley.

Dominic Widdows. 2003. Unsupervised methods for developing taxonomies by combining syntactic and statistical information. In Proceedings of HLT$N A A C L$.

Ichiro Yamada, Kentaro Torisawa, Jun'ichi Kazama, Kow Kuroda, Masaki Murata, Stijn De Saeger, Francis Bond, and Asuka Sumida. 2009. Hypernym discovery based on distributional similarity and hierarchical structures. In Proceedings of EMNLP.

Hui Yang and Jamie Callan. 2009. A metric-based framework for automatic taxonomy induction. In Proceedings of ACL-IJCNLP. 